\title{
BMJ Open Prenatal exposure to antiepileptic drugs and use of primary healthcare during childhood: a population-based cohort study in Denmark
}

\author{
Anne Mette Würtz, ${ }^{1,2}$ Dorte Rytter, ${ }^{2}$ Claus Høstrup Vestergaard, ${ }^{1}$ \\ Jakob Christensen, ${ }^{3}$ Mogens Vestergaard, ${ }^{1,4}$ Bodil Hammer Bech ${ }^{1,2}$
}

To cite: Würtz AM, Rytter D, Vestergaard $\mathrm{CH}$, et al. Prenatal exposure to antiepileptic drugs and use of primary healthcare during childhood: a populationbased cohort study in Denmark. BMJ Open 2017;6: e012836. doi:10.1136/ bmjopen-2016-012836

- Prepublication history for this paper is available online. To view these files please visit the journal online (http://dx.doi.org/10.1136/ bmjopen-2016-012836)

Received 26 May 2016 Revised 15 September 2016 Accepted 13 October 2016

CrossMark

\begin{abstract}
${ }^{1}$ Research Unit for General Practice, Department of Public Health, Aarhus University, Aarhus, Denmark ${ }^{2}$ Section for Epidemiology, Department of Public Health, Aarhus University, Aarhus, Denmark

${ }^{3}$ Department of Neurology, Aarhus University Hospital, Aarhus, Denmark ${ }^{4}$ Section for General Medical Practice, Department of Public Health, Aarhus University, Aarhus, Denmark
\end{abstract}

Correspondence to Anne Mette Lund Würtz; amlw@ph.au.dk

\section{ABSTRACT}

Objective: Prenatal exposure to antiepileptic drugs (AEDs) has been associated with adverse outcomes in the offspring such as congenital malformations and neuropsychiatric disorders. The objective of this study was to investigate whether prenatal exposure to AEDs is also associated with more frequent use of primary healthcare during childhood.

Design: Population-based cohort study.

Setting: Nationwide national registers in Denmark. Participants: All live-born singletons in Denmark during 1997-2012 identified in the Danish National Patient Register and followed until 31 December 2013 $(n=963010)$. Information on prenatal exposure to AEDs for maternal indication of epilepsy and other neurological conditions was obtained from the Danish Register of Medicinal Product Statistics.

Main outcome measures: The primary outcome measure was the number and type of contacts with the general practitioner (GP), excluding routine well-child visits and vaccinations. The secondary outcome measure was specific services provided at the GP contact. The association between prenatal exposure to AEDs and contacts with the GP was estimated by using negative binomial regression adjusting for sex and date of birth of the child, maternal age, cohabitation status, income, education, substance abuse, depression, severe psychiatric disorders and use of antipsychotics, antidepressants and insulin.

Results: Children exposed prenatally to AEDs $(\mathrm{n}=4478)$ had $3 \%(95 \% \mathrm{Cl} 0$ to $5 \%)$ more GP contacts during the study period than unexposed children. This was primarily accounted for by the number of phone contacts. Within each year of follow-up, exposed children tended to have more contacts than unexposed children, but the differences were small. We found no difference between exposed and unexposed children with regard to specific services provided at the GP contact. For the individual AEDs, we found that exposure to valproate or oxcarbazepine was associated with more GP contacts.

Conclusions: We found only minor differences between prenatally AED-exposed and unexposed children in the number of GP contacts.

\section{Strengths and limitations of this study}

- Population-based study including all live-born singletons in Denmark from 1997 to 2012.

- Low risk of selection and information bias due to complete follow-up and high data quality.

- Possibility to adjust for many potential confounders with yearly updated information through linkage with a variety of national registers.

- The study was not able to include information on maternal lifestyle during pregnancy and therefore cannot exclude residual and unknown confounding.

\section{INTRODUCTION}

Epilepsy is the most common neurological disorder that requires medical treatment during pregnancy. ${ }^{1}$ The prevalence of antiepileptic drug (AED) use during pregnancy has increased in recent years in the USA, mainly due to an increase in the use of newer types of AEDs. ${ }^{2}$ The same tendency is seen in Denmark, where the number of pregnant women treated with AEDs has increased slightly during the past decade. This rise is primarily a consequence of increasing use of new types of AEDs, whereas older types are now used less. ${ }^{3}$

Prenatal AED exposure has been associated with adverse health outcomes, such as congenital malformations, ${ }^{4}$ impaired neurodevelopment and cognitive function, ${ }^{5}$ low Apgar score, ${ }^{6}$ enamel defects of the teeth, ${ }^{7}$ risk of autism spectrum disorders and childhood autism ${ }^{3}$ and behavioural problems in preschool children. ${ }^{8}$ Among the specific AEDs, valproate has been associated with a considerably higher risk of birth defects and more consistent adverse neurodevelopmental outcomes in the offspring than other AEDs. ${ }^{5}{ }^{9}$ Prenatal lamotrigine exposure has been associated with low rates of major 
malformations, ${ }^{10-12}$ but studies on newer generations of AEDs (eg, levetiracetam) are still emerging. ${ }^{9}$ Despite accumulating evidence of adverse effects of prenatal AED exposure on birth outcomes and neurodevelopment, little is known about the overall health of children exposed to AEDs in fetal life.

In Denmark, access to general practitioners (GPs) is free of charge and $98 \%$ of the population is registered with a specific general practice. The GPs serve as gatekeepers to the rest of the healthcare system, and all citizens must first contact the GP for all non-emergency symptoms and diseases. ${ }^{13}$ Since most diseases are diagnosed and treated in primary healthcare, the usage of services provided by the GPs may be an important proxy for the overall health of the citizens. The wage of the GPs is funded by the Danish tax system, and the GPs are remunerated according to a combination of capitation and fee-for-service. Consequently, the GPs must carefully register each specific contact and medical procedure in order to receive payment for the services provided. ${ }^{14}$

The aim of this study was to investigate whether AED exposure in fetal life is associated with use of primary healthcare in childhood as measured by number of GP contacts. If prenatal AED exposure increases the overall morbidity, we would expect exposed children to have more GP contacts than unexposed children.

\section{METHODS}

\section{Study design and population}

This population-based cohort study included all singleton live-born children in Denmark from 1 January 1997 to 31 December 2012. Live births were identified from the Danish National Patient Register using code DZ38* of the International Classification of Diseases (ICD), 10th revision. ${ }^{15}$ Every citizen in Denmark is assigned a unique personal identification number in the Danish Civil Registration System ${ }^{16}$ at birth. Using the child's unique personal identification number, we were able to identify the mother in the Danish Civil Registration System. In total, 1019645 children were born in Denmark from 1 January 1997 to 31 December 2012. We excluded 56498 children because they were not registered in the Danish Civil Registration System $(n=5032)$, they could not be linked to their mother $(n=4138)$, they were not singleton $(n=41713)$ or they had missing information on gestational age $(n=5752)$; this left us with 963010 eligible children for inclusion in the present study.

\section{Exposure}

Information on the mother's AED use during pregnancy was obtained from the Danish Register of Medicinal Product Statistics, which holds records of all prescriptions redeemed since 1 January $1996 .{ }^{17}$ However, treatment with AEDs during inpatient hospital admission is not recorded. In this study, AED use was defined as any prescription redeemed with the Anatomical Therapeutic
Chemical (ATC) codes N03A (AEDs) or N05BA09 (clobazam) as defined by the WHO. ${ }^{18}$ The exposure window was defined as 30 days before the estimated first day of the last menstrual period of the mother to the day before the birth. The cumulative dose of AEDs used during the exposure window was calculated from the date of prescription redemption and the dose and packing size of each prescription. The estimated average daily dose of AEDs was calculated by dividing the cumulative dose by the number of days in the exposure window. Based on the defined daily dose, ${ }^{19}$ the estimated daily dose was dichotomised into low $(\leq 50 \%$ of the defined daily dose) and high ( $>50 \%$ of defined daily dose) dose. Among the 963010 children included in the study, 4478 were prenatally exposed to AEDs and 958532 were not exposed.

\section{Outcome}

The primary outcome of interest was the number and type of contacts with the GP after birth in the period from 1 January 1997 to 31 December 2013. The secondary outcome was the children's use of specific GP services provided at the GP contact in the same period. The GPs register every specific contact and health-related service provided for each citizen by using the unique personal identification number, and the information is recorded electronically in the National Health Insurance Service Register. ${ }^{20}$ From this register, we obtained information on number of consultations in daytime and out-of-hours, telephone and email contacts, and diagnostic tests performed during daytime. We excluded contacts forming part of routine well-child visits and the Danish childhood vaccination programme. In Denmark, three well-child visits are scheduled during the first year of life and then one per year up to the age of 5 years. The specific outcomes of the study were the total number of GP contacts per year (labelled 'All contacts'), the number of GP visits, daytime GP visits, out-of-hours GP visits, telephone contacts and specific activity codes to examine the reasons for the contacts. The outcome measure 'All contacts' included daytime visits, out-of-hours visits, telephone contacts, email contacts and home visits. Telephone contacts included telephone contacts during both daytime and out-of-hours. Among the specific services provided at the GP contacts, we only included activities related to physical health and these were blood sample (activity codes 2601 and 2101), B-haemoglobin (activity code 7108), peak flow/spirometry (activity codes 7113, 7121 and 7183), urinary stix (activity code 7101), strep-A test (ie, rapid strep test) (activity code 7109) and $\mathrm{C}$ reactive protein (activity code $7120)$.

\section{Covariates}

Information on sex of the children and maternal age was obtained from the Danish Civil Registration System. Information on gestational age, date of birth and Apgar score at 5 min of the children was obtained from the 
Danish National Patient Register. ${ }^{15}$ Information on maternal cohabitation status, income and education was obtained from Statistics Denmark. ${ }^{21}$ Mothers diagnosed with epilepsy (ICD-8 code 345 and ICD-10 codes G40

Table 1 Characteristics of the children and their mothers at birth of the index child; mean (SD) or proportion*

\begin{tabular}{|c|c|c|}
\hline & $\begin{array}{l}+A E D \\
(n=4478)\end{array}$ & $\begin{array}{l}\text {-AED } \\
(n=958532)\end{array}$ \\
\hline \multicolumn{3}{|l|}{ Characteristics of the children } \\
\hline \multicolumn{3}{|l|}{ Sex, \% } \\
\hline Female & 47 & 49 \\
\hline Male & 53 & 51 \\
\hline \multicolumn{3}{|l|}{ Gestational age } \\
\hline Mean (SD), days & $276(14)$ & $279(13)$ \\
\hline Preterm (<37 weeks), \% & 8 & 5 \\
\hline Term ( $\geq 37$ weeks), \% & 92 & 95 \\
\hline \multicolumn{3}{|l|}{ Birth year, \% } \\
\hline $1997-2001$ & 28 & 32 \\
\hline 2002-2006 & 28 & 31 \\
\hline 2007-2012 & 44 & 36 \\
\hline \multicolumn{3}{|l|}{ Apgar score at $5 \mathrm{~min}, \%$} \\
\hline $0-7$ & 2 & 1 \\
\hline $8-10$ & 98 & 98 \\
\hline Missing & $<1$ & $<1$ \\
\hline \multicolumn{3}{|l|}{ Maternal characteristics } \\
\hline \multicolumn{3}{|l|}{ Maternal age, years } \\
\hline Mean (SD) & $30(5)$ & $30(5)$ \\
\hline$<21, \%$ & 2 & 2 \\
\hline $21-25, \%$ & 13 & 12 \\
\hline $26-30, \%$ & 33 & 34 \\
\hline $31-35, \%$ & 34 & 36 \\
\hline$\geq 36, \%$ & 19 & 17 \\
\hline \multicolumn{3}{|l|}{ Cohabitation, \% } \\
\hline Married & 51 & 60 \\
\hline Cohabiting & 35 & 33 \\
\hline Single & 13 & 7 \\
\hline Missing & $<1$ & $<1$ \\
\hline \multicolumn{3}{|l|}{ Income, \% } \\
\hline Quartile 1 & 31 & 25 \\
\hline Quartile 2 & 30 & 25 \\
\hline Quartile 3 & 23 & 25 \\
\hline Quartile 4 & 16 & 25 \\
\hline Missing & $<1$ & $<1$ \\
\hline \multicolumn{3}{|l|}{ Education, \% } \\
\hline$<10$ years & 32 & 18 \\
\hline $10-15$ years & 41 & 45 \\
\hline$>15$ years & 24 & 33 \\
\hline Missing & 2 & 3 \\
\hline \multicolumn{3}{|l|}{ Diagnoses, \% } \\
\hline Epilepsy & 72 & 1 \\
\hline Substance abuse & 9 & 3 \\
\hline Depression & 11 & 2 \\
\hline Severe psychiatric disorders & 5 & $<1$ \\
\hline \multicolumn{3}{|l|}{ Concurrent drug use, $\%$} \\
\hline Antipsychotics & 2 & $<1$ \\
\hline Antidepressants & 15 & 2 \\
\hline Insulin & 1 & 1 \\
\hline
\end{tabular}

and G41) before the end of the index pregnancy were identified from the Danish National Patient Register. ${ }^{15}$ The Danish National Patient Register includes information on all inpatients from 1978 and all outpatients from 1995 onwards. $^{15}$ In the Danish Psychiatric Central Research Register, ${ }^{22}$ we identified mothers with a diagnosis of substance abuse (ICD-8 codes 291, 294.3, 303 and 304 and ICD-10 codes F10-F19), depression (ICD-8 codes 296.0, 298.0 and 300.4 and ICD-10 codes F32-F33) and severe psychiatric disorders (ICD-8 codes 295, 296.1-296.8 and 298.1 and ICD-10 codes F20 and F30-F31) before the end of the index pregnancy. The Danish Psychiatric Central Research Register includes information on all inpatients from 1969 and outpatients from 1995 onwards. $^{22}$ Information on maternal use of antipsychotics (ATC code N05A), antidepressants (ATC code N06A) and insulin (ATC code A10A) during the exposure window was obtained from the Danish Register of Medicinal Product Statistics.

\section{Data analysis}

The children were followed from date of birth until death, emigration or 31 December 2013, whichever occurred first. The first day of the last menstrual period of the mother was estimated by subtracting the gestational age from the date of birth of the child.

The characteristics of the eligible children and their mothers were summarised using means and SDs or proportions. Incidence rate ratios (IRR) and the corresponding $95 \%$ CIs for both the entire follow-up period and each year of follow-up were estimated using a negative binomial regression model applying cluster robust variance to account for correlations in the children's use of GP services due to multiparity in the mothers. The IRRs were adjusted for sex (male, female) and date of birth of the children (date; continuous) and for

Table 2 IRR (95\% Cl) for GP contact by type of contact comparing children with prenatal AED exposure to unexposed children

\begin{tabular}{lll}
\hline Type of contact & $\begin{array}{l}\text { Crude IRR } \\
(\mathbf{9 5 \%} \mathbf{C l})\end{array}$ & $\begin{array}{l}\text { Adjusted IRR } \\
\mathbf{( 9 5 \% ~} \mathbf{C l})^{\star}\end{array}$ \\
\hline All contacts & $1.17(1.14$ to 1.20$)$ & $1.03(1.00$ to 1.05$)$ \\
Visits & $1.14(1.11$ to 1.16$)$ & $1.01(0.99$ to 1.04$)$ \\
$\quad$ Daytime visits & $1.13(1.10$ to 1.15$)$ & $1.02(1.00$ to 1.05$)$ \\
$\quad \begin{array}{l}\text { Out-of-hours } \\
\text { visits }\end{array}$ & $1.18(1.14$ to 1.23$)$ & $0.96(0.93$ to 1.00$)$ \\
Phone contacts & $1.22(1.18$ to 1.25$)$ & $1.04(1.01$ to 1.08$)$ \\
\hline
\end{tabular}

IRRs and $95 \% \mathrm{Cls}$ were estimated using negative binomial regression applying cluster robust variance to account for correlations in the children's use of GP services due to multiparity in the mothers.

*Adjusted for sex and date of birth of the children and maternal age, cohabitation status, income, education, diagnosis of substance abuse, depression and severe psychiatric disorders as well as use of antipsychotics, antidepressants and insulin. AED, antiepileptic drug; GP, general practitioner; IRR, incidence rate ratio. 
maternal age (years; continuous), cohabitation status (married, cohabiting, single, missing), income (quartiles by year and missing category), education ( $<10$ years, 10 15 years, $>15$ years, missing), diagnosis of substance abuse (yes, no), depression (yes, no) and severe psychiatric disorders (yes, no) as well as use of antipsychotics (yes, no), antidepressants (yes, no) and insulin (yes, no) at some point during the index pregnancy. The variables maternal cohabitation status, income, education, diagnosis of substance abuse, depression and severe psychiatric disorders were included as time-varying covariates.

To investigate potential confounding by indication, we stratified the analyses for the primary outcome according to 'never' versus 'ever' diagnosis of epilepsy of the mother registered in the Danish National Patient Register before the end of the index pregnancy. This stratified analysis was additionally carried out using a cut-off value for epilepsy diagnosis of 5 years prior to the first day of the last menstrual period before the index pregnancy.
Besides the investigation of all AEDs as one group, we studied the six most commonly used AEDs separately. We furthermore investigated the association with GP contacts for low and high estimated daily doses of the six most commonly used AEDs (monotherapy only) in addition to usage of AEDs as monotherapy or polytherapy.

All statistical analyses were performed in Stata V.13 (StataCorp LP, Texas, USA).

\section{RESULTS}

We followed 963010 children for a median of 9 years (range 1-16 years). Children prenatally exposed to AEDs were more likely to be male and to be born preterm than unexposed children (table 1). Furthermore, exposed children were more likely to be born to mothers living alone, with short education, low income, a diagnosis of substance abuse, depression or
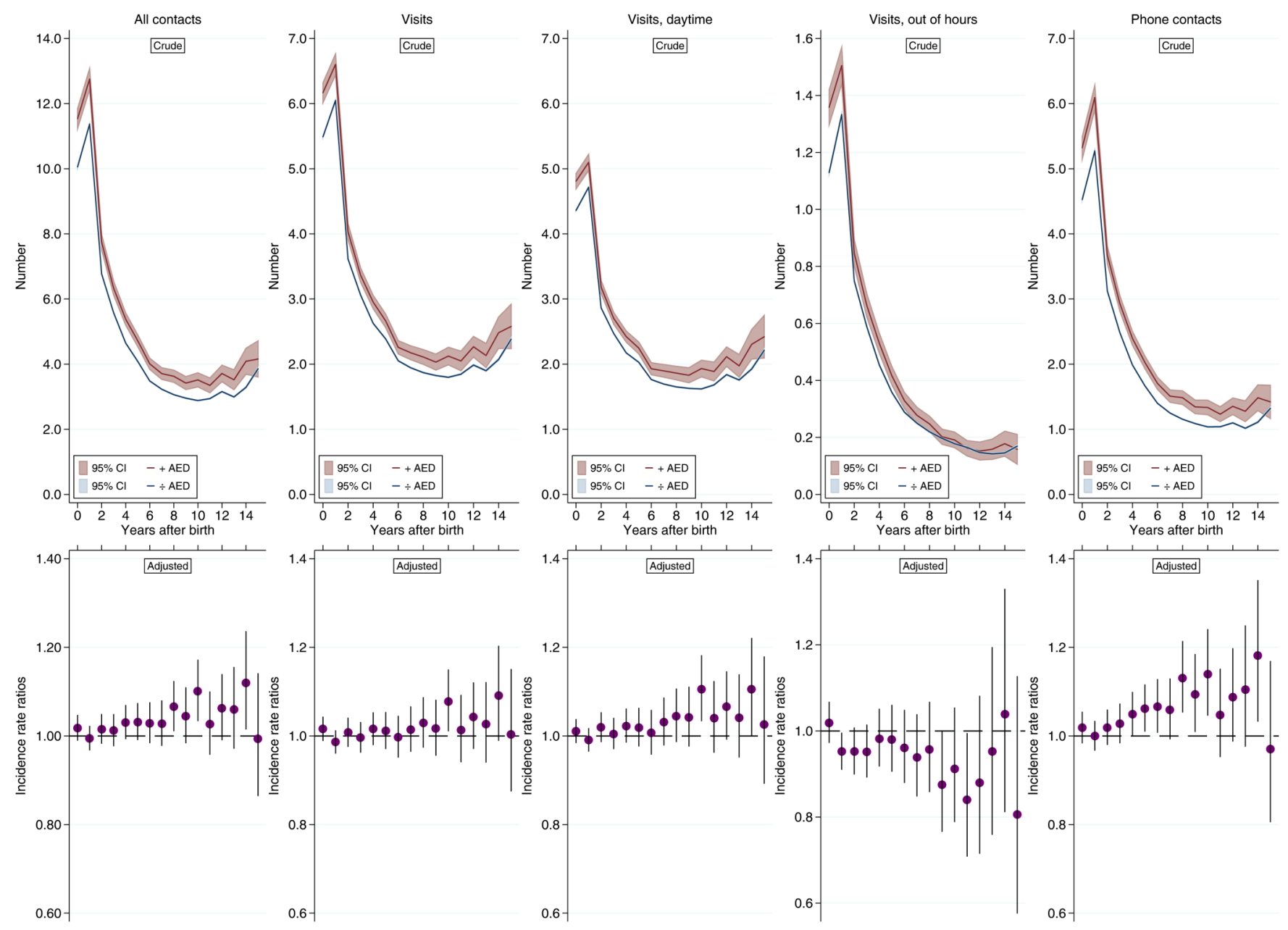

Figure 1 Type of GP contact by age of the children in 1-year intervals; unadjusted mean number of contacts in top panels and adjusted IRRs $(95 \% \mathrm{Cl})$ in bottom panels. IRRs and $95 \%$ Cls were estimated using negative binomial regression applying cluster robust variance to account for correlations in the children's use of GP services due to multiparity in the mothers. Adjusted for sex and date of birth of the children and for maternal age, cohabitation status, income, education, diagnosis of substance abuse, depression and severe psychiatric disorders as well as use of antipsychotics, antidepressants and insulin. GP, general practitioner; IRR, incidence rate ratio. 
severe psychiatric disorders and concurrent use of antipsychotics, antidepressants or insulin.

The total number of GP contacts during the entire follow-up period was 46736112 . The mean number of GP contacts among unexposed children was 4.6 contacts per year. Throughout the entire study period, children who had been prenatally exposed to AEDs had 3\% more contacts with the GP than unexposed children (table 2); the adjusted IRR for all contacts was 1.03 (95\% CI 1.00 to 1.05$)$.

Figure 1 shows the mean number of contacts and adjusted IRRs (95\% CI) by type of contact within 1-year intervals from the date of birth. Overall, the annual number of GP contacts was similar among exposed and unexposed children after adjustment for covariates, although exposed children tended to have more frequent contacts in later childhood. A similar picture was found for total number of visits, daytime visits and telephone contacts, but not for out-of-hours visits. When investigating the specific services provided in connection with the contacts (figure 2), we found that the use of peak flow/spirometry tests and biological examinations was similar among exposed and unexposed children. However, fewer strep-A tests tended to be performed among exposed children compared to unexposed children.

When stratifying the analyses for the primary outcome by maternal diagnosis of epilepsy as registered in the Danish National Patient Register before the end of the index pregnancy, no difference between the strata was found (figure 3). However, among children born to a mother with no diagnosis of epilepsy, we found a tendency towards more GP contacts by phone among prenatally AED-exposed children compared to unexposed children. Restricting the maternal diagnosis of epilepsy to the 5-year period prior to the index pregnancy showed similar results.

The six most commonly used drugs among women holding prescriptions for AEDs were: clonazepam $(10 \%)$, oxcarbazepine $(11 \%)$, lamotrigine $(44 \%)$, carbamazepine $(12 \%)$, valproate $(14 \%)$ and levetiracetam $(4 \%)$. Throughout the entire study period, children
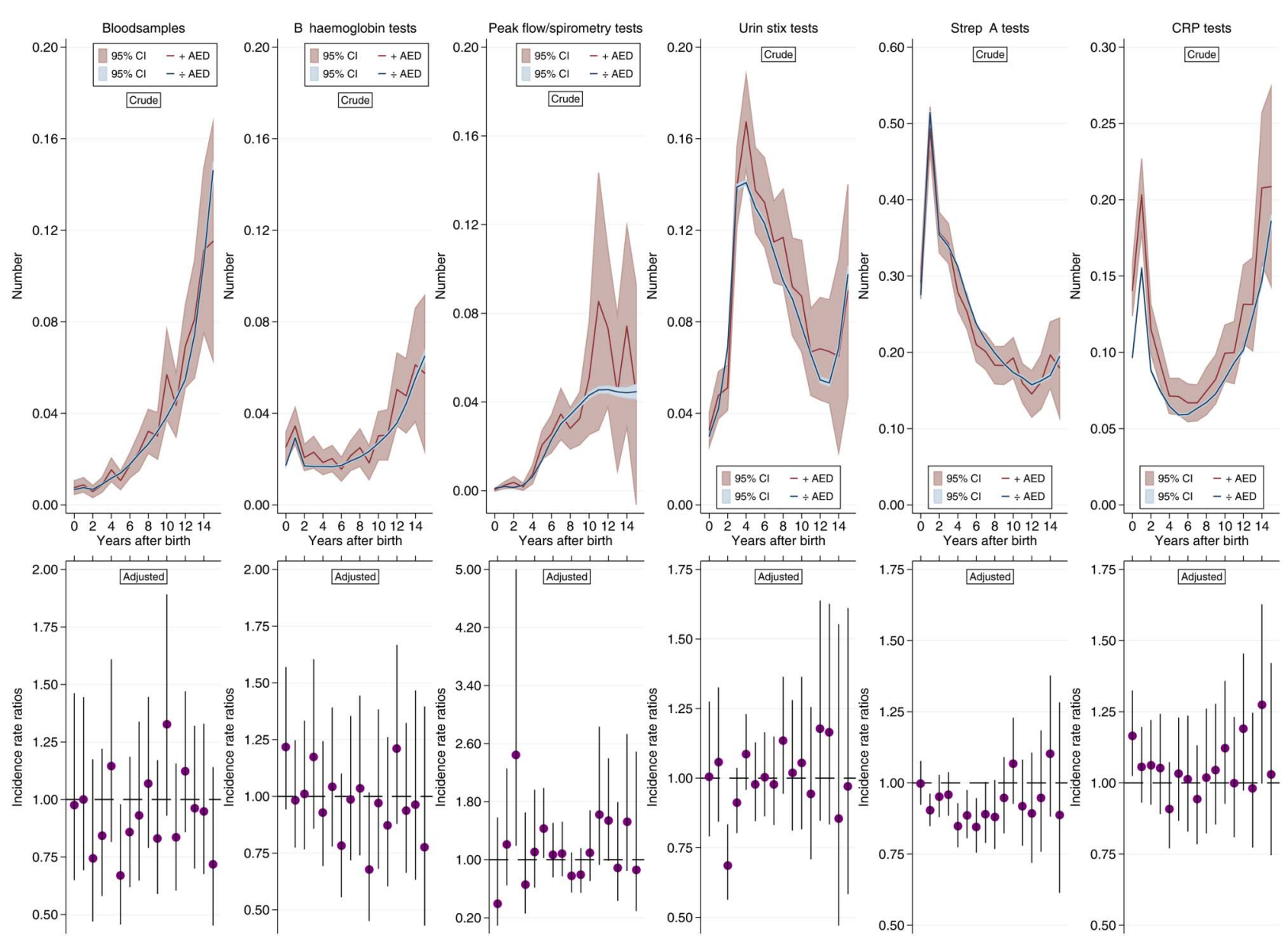

Figure 2 Services provided at the GP contacts by age of the children in 1-year intervals; unadjusted mean number of contacts in top panels and adjusted IRRs $(95 \% \mathrm{Cl}$ ) in bottom panels. IRRs and $95 \%$ Cls were estimated using negative binomial regression applying cluster robust variance to account for correlations in the children's use of GP services due to multiparity in the mothers. Adjusted for sex and date of birth of the children and for maternal age, cohabitation status, income, education, diagnosis of substance abuse, depression and severe psychiatric disorders as well as use of antipsychotics, antidepressants and insulin. GP, general practitioner; IRR, incidence rate ratio. 
exposed to oxcarbazepine or valproate had more GP contacts than children unexposed to any AED (table 3). Adjusted IRRs were 1.08 (95\% CI 1.00 to 1.15) for oxcarbazepine and 1.08 (95\% CI 1.01 to 1.15 ) for valproate.
Within 1-year intervals, exposure to oxcarbazepine, valproate or levetiracetam tended to be associated with more GP contacts, although not statistically significant, whereas no difference was found in number of GP
All contacts
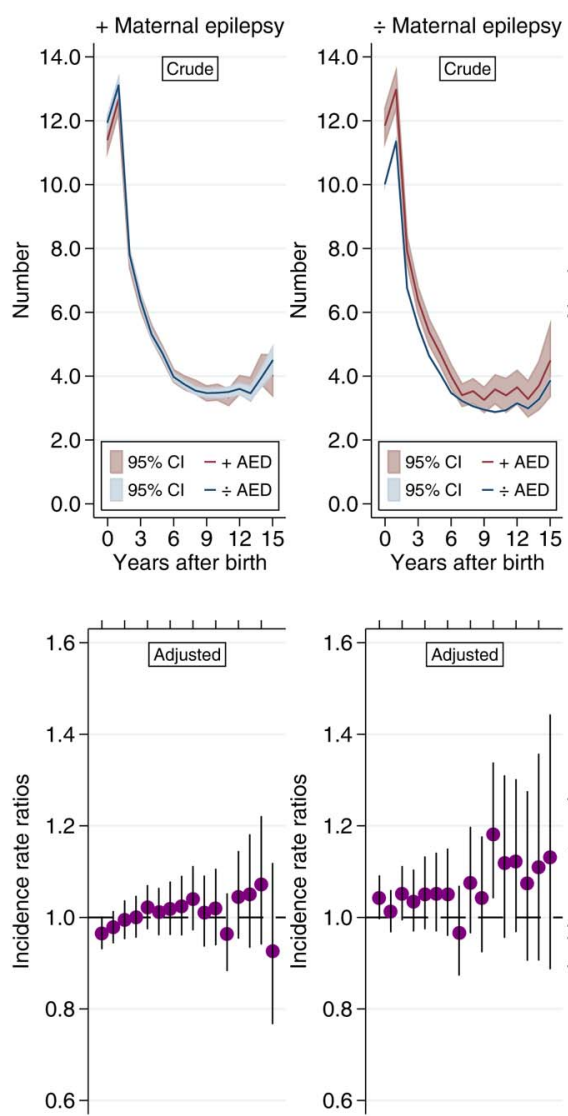

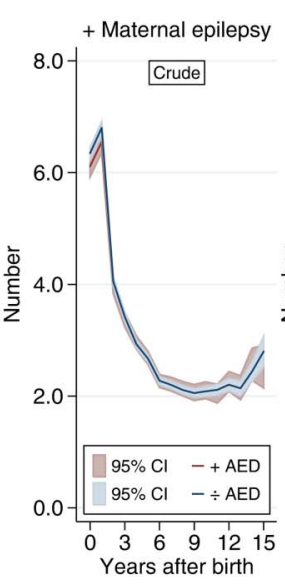

Visits

Phone contacts
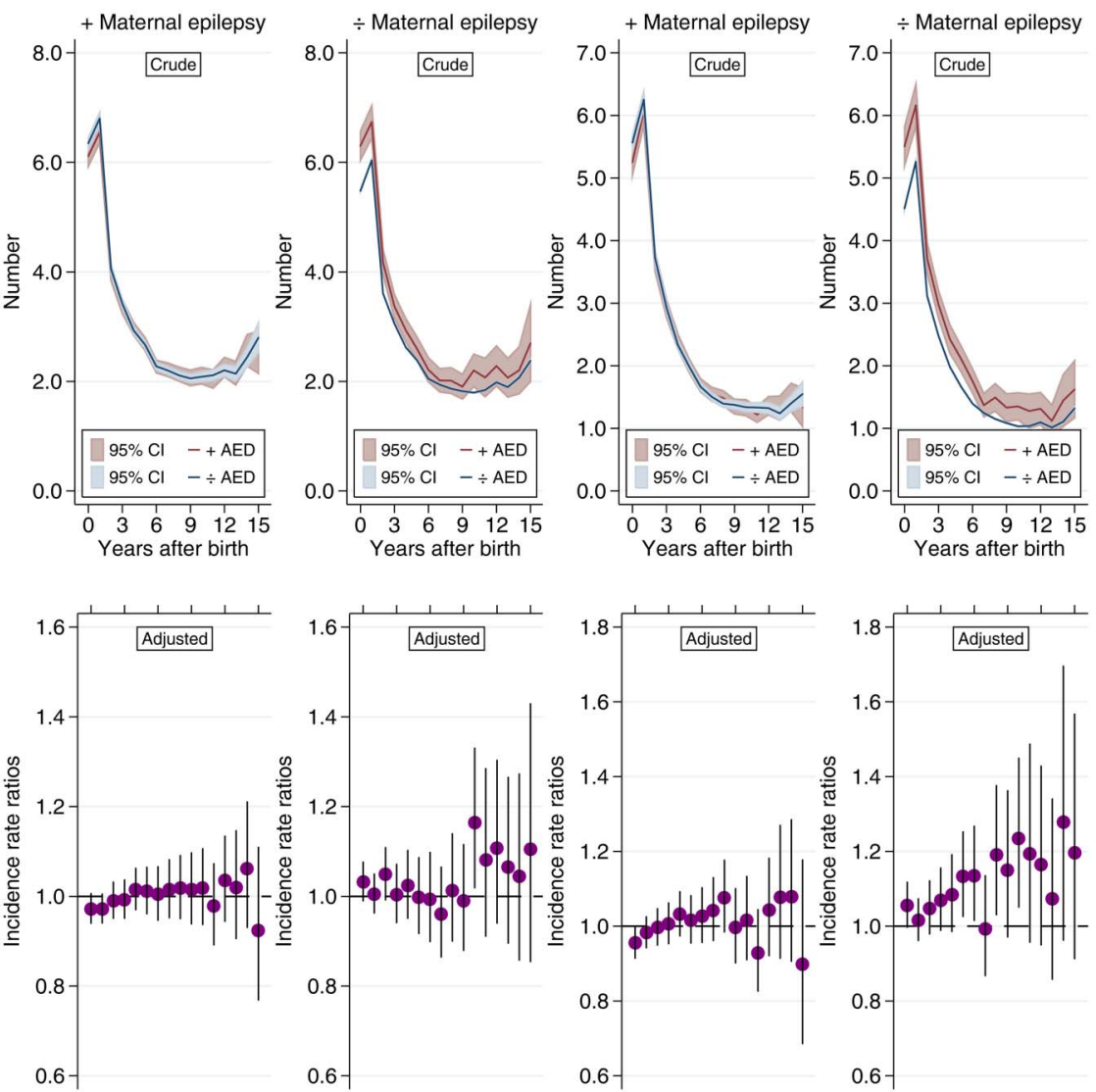

Figure 3 Type of GP contact stratified according to maternal diagnosis of epilepsy at the index pregnancy by age of the children in 1 -year intervals; unadjusted mean number of contacts in top panels and adjusted IRRs $(95 \% \mathrm{Cl})$ in bottom panels. During 15098 pregnancies with a maternal diagnosis of epilepsy, 3240 mothers used AEDs, and during 947912 pregnancies with no maternal diagnosis of epilepsy, 1238 mothers used AEDs. IRRs and $95 \%$ Cls were estimated using negative binomial regression applying cluster robust variance to account for correlations in the children's use of GP services due to multiparity in the mothers. Adjusted for sex and date of birth of the children and for maternal age, cohabitation status, income, education, diagnosis of substance abuse, depression and severe psychiatric disorders as well as use of antipsychotics, antidepressants and insulin. AEDs, antiepileptic drugs; GP, general practitioner; IRR, incidence rate ratio.

Table 3 Type of AED and IRR $(95 \% \mathrm{Cl})$ for any GP contact comparing exposed children to children unexposed to any AED

\begin{tabular}{lccc}
\hline Type of AED & $\mathbf{n}$ & Crude IRR (95\% CI) & Adjusted IRR (95\% CI)* \\
\hline Clonazepam & 465 & $1.07(1.00$ to 1.15$)$ & $0.97(0.90$ to 1.04$)$ \\
Oxcarbazepine & 511 & $1.14(1.07$ to 1.22$)$ & $1.08(1.00$ to 1.15$)$ \\
Lamotrigine & 1977 & $1.27(1.22$ to 1.31$)$ & $0.99(0.95$ to 1.03$)$ \\
Carbamazepine & 516 & $0.97(0.91$ to 1.04$)$ & $0.96(0.90$ to 1.04$)$ \\
Valproate & 610 & $1.16(1.09$ to 1.23$)$ & $1.08(1.01$ to 1.15$)$ \\
Levetiracetam & 196 & $1.69(1.49$ to 1.91$)$ & $1.08(0.95$ to 1.23$)$ \\
\hline IRRs and 95\% Cls were estimated using negative binomial regression applying cluster robust variance to account for correlations in the \\
children's use of GP services due to multiparity in the mothers. The number of children unexposed to any AED is 958 532. \\
*Adjusted for sex and date of birth of the children and maternal age, cohabitation status, income, education, diagnosis of substance abuse, \\
depression and severe psychiatric disorders as well as use of antipsychotics, antidepressants and insulin. \\
AED, antiepileptic drug; GP, general practitioner; IRR, incidence rate ratio.
\end{tabular}


contacts for children exposed to clonazepam, lamotrigine or carbamazepine compared to children unexposed to any AED (figure 4).

Looking into the AED dose (figure 5), a high estimated daily dose of valproate or levetiracetam was associated with more GP contacts. For oxcarbazepine, low estimated daily dose was associated with a statistically significant higher risk of GP contact. Exposure to valproate as monotherapy (figure 6) was associated with a statistically significant higher risk of GP contact, whereas a statistically non-significant tendency for levetiracetam monotherapy exposure was observed.

\section{DISCUSSION}

In this population-based follow-up study, we investigated the number of GP contacts among prenatally AED-exposed and unexposed children. GP contacts could be a proxy for the health status of the included children, although more severe disease would not be captured as a GP contact prompting longer hospitalisation and potential follow-up at the hospital might count as only one contact. Unfortunately, information on the reasons for the GP contacts and the children's condition and potential diagnoses given at the GP contact is not available in the National Health Insurance Service Register. We found that prenatally exposed children had only slightly more GP contacts than unexposed children and few estimates were statistically significant. Stratifying the analyses by maternal diagnosis of epilepsy showed overall similar results for children born to mothers with and without epilepsy. However, the tendency towards more phone contacts for exposed children born to mothers without a diagnosis of epilepsy could indicate confounding by indication from other neurological or mental disorders that are treated with AEDs, such as bipolar disorder. Mothers with such a diagnosis could be prone to worry more than other mothers and thus might contact the GP more often. ${ }^{23}$
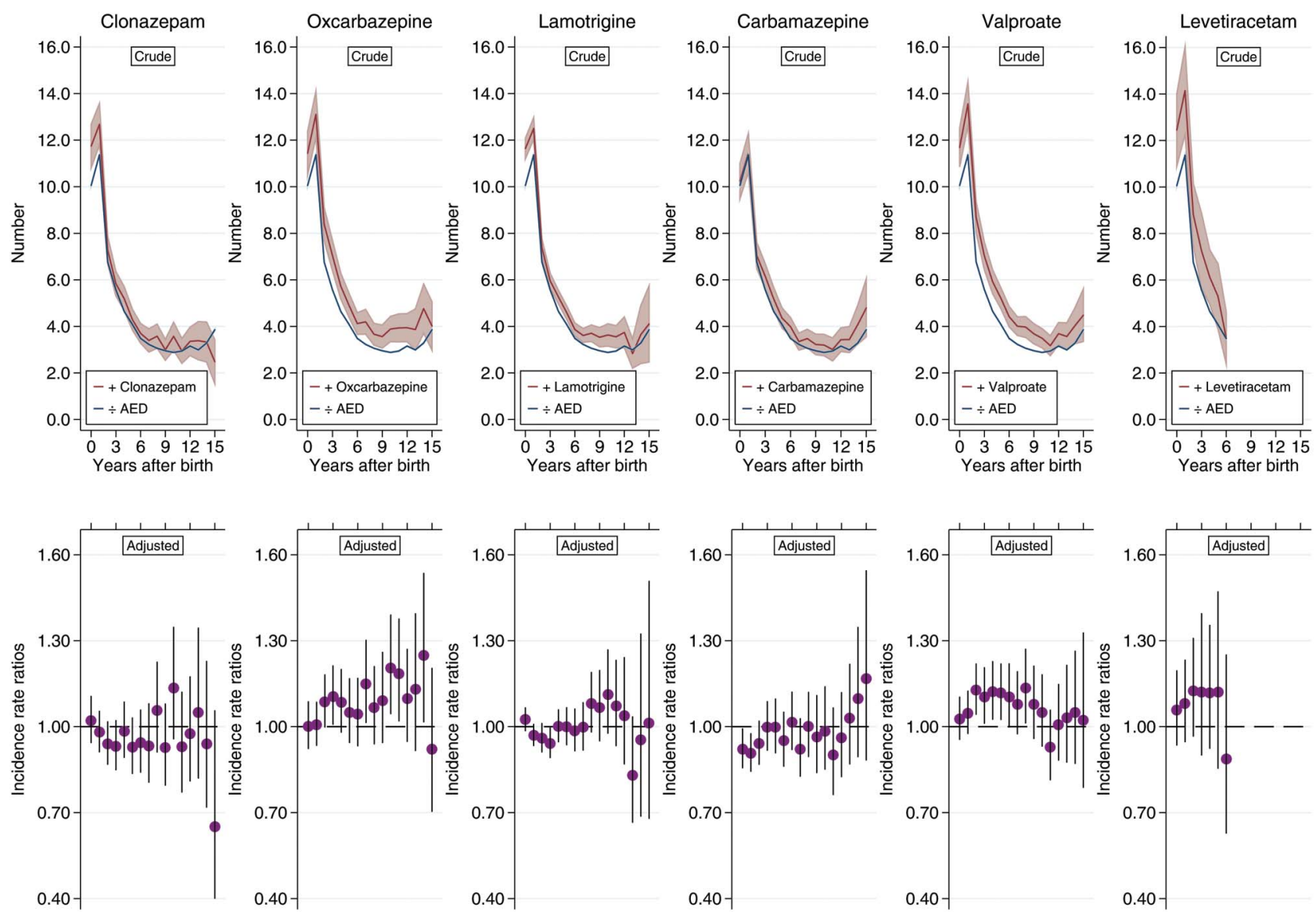

Figure 4 Type of AED and risk of any GP contact by age of the children in 1-year intervals compared to children unexposed to any AED ( $n=958$ 532); unadjusted mean number of contacts in top panels and adjusted IRRs (95\% Cl) in bottom panels. The number of exposed children for each type of AED is 465 for clonazepam, 511 for oxcarbazepine, 1977 for lamotrigine, 516 for carbamazepine, 610 for valproate and 196 for levetiracetam. IRRs and 95\% Cls were estimated using negative binomial regression applying cluster robust variance to account for correlations in the children's use of GP services due to multiparity in the mothers. Adjusted for sex and date of birth of the children and for maternal age, cohabitation status, income, education, diagnosis of substance abuse, depression and severe psychiatric disorders as well as use of antipsychotics, antidepressants and insulin. AEDs, antiepileptic drugs; GP, general practitioner; IRR, incidence rate ratio. 


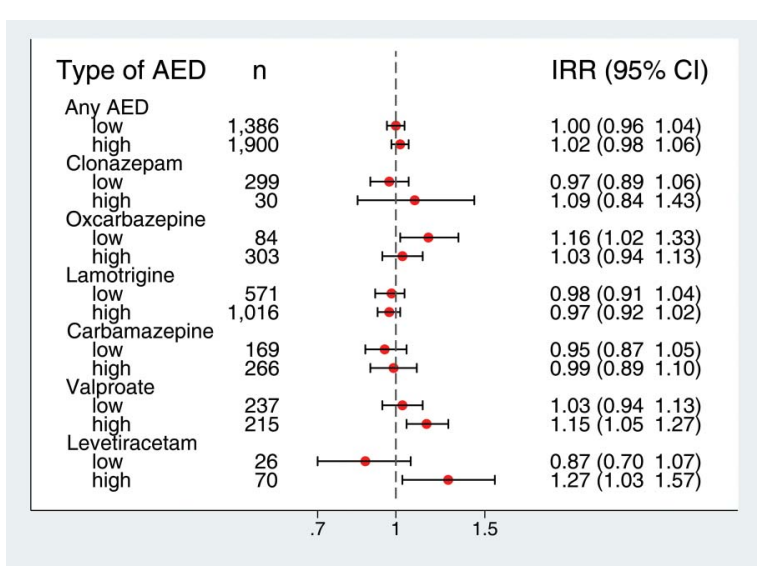

Figure 5 Type and dose of AED (monotherapy only) and risk of any GP contact compared to children unexposed to any AED ( $n=958532)$. The figures indicate the number of children exposed to each type and dose of AED. IRRs and 95\% Cls were estimated using confounder adjusted negative binomial regression. High daily dose for the different AEDs was defined as: clonazepam $>4 \mathrm{mg} /$ day, oxcarbazepine $>500 \mathrm{mg} /$ day, lamotrigine $>150 \mathrm{mg} /$ day, carbamazepine $>500 \mathrm{mg} /$ day, valproate $>750 \mathrm{mg} /$ day and levetiracetam $>750 \mathrm{mg} /$ day. The figure for any AED is the sum of the six most commonly used AEDs. AED, antiepileptic drug; GP, general practitioner; IRR, incidence rate ratio.

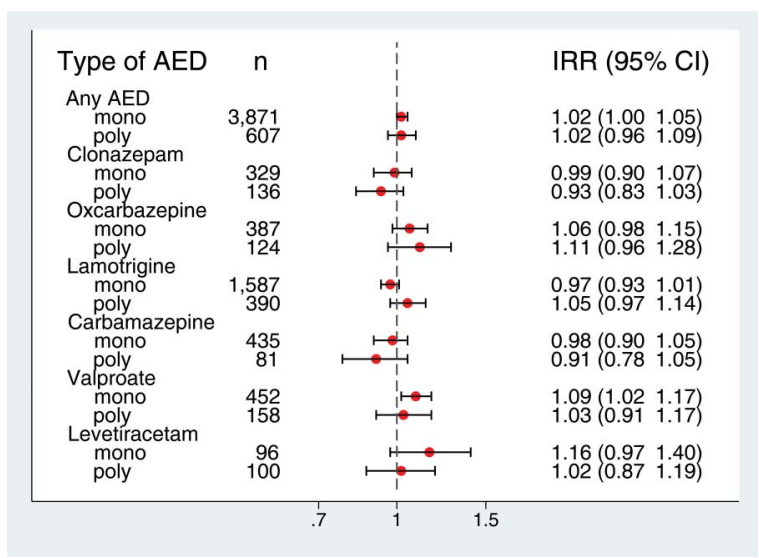

Figure 6 Type of AED, usage as monotherapy or polytherapy and risk of any GP contact compared to children unexposed to any AED ( $n=958532)$. The figures indicate the number of children exposed to each type and usage of AED. Monotherapy is defined as exposure to only the specific AED given, and polytherapy is defined as exposure to the specific AED in combination with one or more other AEDs. IRRs and 95\% Cls were estimated using confounder adjusted negative binomial regression. AED, antiepileptic drug; GP, general practitioner; IRR, incidence rate ratio.

All live-born singletons in Denmark from 1997 to 2012 were included in the study, and the follow-up of the children was nearly complete, which limits the concern for selection bias. The information on fetal AED exposure was identified from nationwide register information on redemptions for maternal AED prescriptions before and during pregnancy. Random misclassification of the AED exposure cannot be excluded as it is uncertain if all redeemed prescriptions were taken. However, a previous study found high compliance with prescribed AEDs among pregnant Danish women. ${ }^{24}$ Therefore, we expect this potential random misclassification to be a minor issue. The information on the use of GP services was obtained from the National Health Insurance Service Register. Since the unique personal identification number is recorded for each GP contact, the accuracy in the identification of each individual is considered to be high. The completeness of the services registered in the National Health Insurance Service Register is also assumed to be high because the remuneration of the GPs depends on accurate registration of each individual contact and medical procedure. ${ }^{20}$ Furthermore, the invoices written by the GPs are checked by spot checks and algorithms that identify GPs with unusual reporting. ${ }^{20}$ Since the information on exposure and outcome is collected separately and as a matter of routine and therefore not depending on self-report, it is unlikely that the registration of GP contacts is influenced by the knowledge of prescriptions redeemed. Hence, information bias is unlikely to have affected our results, but the potential random misclassification could bias the results towards the null. In our analyses, adjustment for potential confounders generally attenuated the risk estimates, which may indicate confounding from these risk factors. Furthermore, confounding from unknown and unmeasured risk factors cannot be excluded. We mostly had sociodemographic and medical information about the mothers. Unfortunately, we were not able to adjust for maternal lifestyle factors like smoking, diet and physical activity during pregnancy, which might influence the general health of the children. An association between prenatal AED exposure and Apgar score at 5 min after birth has been found. ${ }^{6}$ Furthermore, a low Apgar score at birth has been associated with high overall morbidity in childhood including neurological disability, ${ }^{25-27}$ epilepsy $^{28} 29$ and cancer. ${ }^{30}$ To avoid collider bias, we did not adjust for this potential intermediate variable. ${ }^{31}$

We are not aware of other studies investigating the association between prenatal AED exposure and use of primary healthcare in childhood. Another stressful prenatal exposure, maternal loss of a close relative, has been associated with greater use of primary healthcare; this has mainly been due to contacts related to mental health and infections. ${ }^{32}$ In this study, we were not able to investigate the association with mental health, because the children were followed only up to the age of a maximum 16 years. When investigating the different AED types separately, we found that exposure to oxcarbazepine or valproate was associated with a statistically significantly higher risk of GP contact. We found no association between exposure to lamotrigine, the most commonly used AED for pregnant women in Denmark, and risk of GP contact. In analyses of the AED dose, a high dose of valproate or levetiracetam was associated 
with more GP contacts. Our findings are in accordance with previous studies, which found prenatal valproate exposure to be associated with high risk of poor neurodevelopment in infancy and childhood. ${ }^{33}$ Furthermore, a higher risk of adverse outcomes in the children has been found for higher doses of valproate exposure. ${ }^{34} \mathrm{We}$ have no explanation for the finding of a higher risk of GP contact for prenatal exposure to low dose, but not high dose, of oxcarbazepine. Carbamazepine exposure has not been found to be associated with low cognitive functioning, ${ }^{35}$ and data on newer medications such as levetiracetam and lamotrigine are limited. ${ }^{33} 34$

We observed a difference of $3 \%$ in number of GP contacts during the entire follow-up period between prenatally AED-exposed and unexposed children. It can be debated whether this slightly higher risk of GP contact is clinically relevant. The average number of contacts during the entire follow-up period was 4.6 per year among unexposed children; 3\% corresponds to 0.13 more contacts per year or 2.02 contacts during the entire follow-up period. Although prenatal AED exposure has been associated with a number of adverse outcomes in the offspring, for example, congenital malformations and neurodevelopmental disorders, it seems to have only a modest influence on the overall health measured by number of GP contacts during childhood. Our findings are thus generally reassuring for women in need of AED treatment during pregnancy, including women with epilepsy. However, we cannot exclude that the minor difference in number of contacts is caused by a small group of children having very frequent use of the primary healthcare system because of illness. In this particular case, our results would be clinically relevant. Further research could improve our knowledge of the interactions between specific somatic and psychiatric morbidity in an expecting mother and her pharmacotherapy as well as the potential consequences of such interactions for the fetus. Moreover, studies focusing on the effects of the individual drugs are warranted as our results indicated a higher number of GP contacts for some of the investigated drugs.

Contributors MV and BHB initiated the study and obtained the funding. All authors participated in the design of the study. CHV performed the statistical analyses under the guidance of BHB and AMLW. AMLW wrote the manuscript. All authors were involved in the interpretation of data and critical revision of the manuscript. AMLW had the primary responsibility for the final content. All authors read and approved the final manuscript.

Funding This work was supported by the Programme for Clinical Research Infrastructure (PROCRIN) established by the Lundbeck Foundation and the Novo Nordisk Foundation and administered by the Danish Regions.

Competing interests JC has received honoraria for serving on the Scientific Advisory Board of UCB Nordic and Eisai AB. JC has also received honoraria for giving lectures from UCB Nordic and Eisai $A B$ and funding for a trip from UCB Nordic. JC is involved in clinical trials involving the following companies: Pfizer, Novartis, Eisai AB and Sage Therapeutics.

Ethics approval The study was approved by the Danish Data Protection Agency. Ethical approval was not required according to Danish regulations due to the register-based nature of the study.

Provenance and peer review Not commissioned; externally peer reviewed.
Data sharing statement No additional data are available.

Open Access This is an Open Access article distributed in accordance with the Creative Commons Attribution Non Commercial (CC BY-NC 4.0) license, which permits others to distribute, remix, adapt, build upon this work noncommercially, and license their derivative works on different terms, provided the original work is properly cited and the use is non-commercial. See: http:// creativecommons.org/licenses/by-nc/4.0/

\section{REFERENCES}

1. Hauser WA, Annegers JF, Rocca WA. Descriptive epidemiology of epilepsy: contributions of population-based studies from Rochester, Minnesota. Mayo Clin Proc 1996;71:576-86.

2. Bobo WV, Davis RL, Toh S, et al. Trends in the use of antiepileptic drugs among pregnant women in the US, 2001-2007: a medication exposure in pregnancy risk evaluation program study. Paediatr Perinat Epidemiol 2012;26:578-88.

3. Christensen J, Gronborg TK, Sorensen MJ, et al. Prenatal valproate exposure and risk of autism spectrum disorders and childhood autism. JAMA 2013;309:1696-703.

4. Harden $\mathrm{CL}$, Meador KJ, Pennell PB, et al. Management issues for women with epilepsy-Focus on pregnancy (an evidence-based review): II. Teratogenesis and perinatal outcomes: report of the Quality Standards Subcommittee and Therapeutics and Technology Subcommittee of the American Academy of Neurology and the American Epilepsy Society. Epilepsia 2009;50:1237-46.

5. Velez-Ruiz NJ, Meador KJ. Neurodevelopmental effects of fetal antiepileptic drug exposure. Drug Saf 2015;38:271-8.

6. Christensen J, Pedersen HS, Kjaersgaard MI, et al. Apgar-score in children prenatally exposed to antiepileptic drugs: a population-based cohort study. BMJ Open 2015;5:e007425.

7. Jacobsen PE, Henriksen TB, Haubek D, et al. Developmental enamel defects in children prenatally exposed to anti-epileptic drugs. PLOS ONE 2013;8:e58213.

8. Kjaer D, Christensen J, Bech BH, et al. Preschool behavioral problems in children prenatally exposed to antiepileptic drugs-a follow-up study. Epilepsy Behav 2013;29:407-11.

9. Bath KG, Scharfman HE. Impact of early life exposure to antiepileptic drugs on neurobehavioral outcomes based on laboratory animal and clinical research. Epilepsy Behav 2013;26:427-39.

10. Meador KJ, Loring DW. Developmental effects of antiepileptic drugs and the need for improved regulations. Neurology 2016;86:297-306.

11. Tomson T, Battino D, Bonizzoni E, et al. Dose-dependent risk of malformations with antiepileptic drugs: an analysis of data from the EURAP epilepsy and pregnancy registry. Lancet Neurol 2011;10:609-17.

12. Vajda FJ, Graham JE, Hitchcock AA, et al. Is lamotrigine a significant human teratogen? Observations from the Australian Pregnancy Register. Seizure 2010;19:558-61.

13. Christiansen T. Organization and financing of the Danish healthcare system. Health Policy 2002;59:107-18.

14. Pedersen KM, Andersen JS, Sondergaard J. General practice and primary healthcare in Denmark. J Am Board Fam Med 2012;25 (Suppl 1):S34-8.

15. Schmidt M, Schmidt SA, Sandegaard JL, et al. The Danish National Patient Registry: a review of content, data quality, and research potential. Clin Epidemiol 2015;7:449-90.

16. Pedersen CB. The Danish Civil Registration System. Scand J Public Health 2011;39:22-5.

17. Kildemoes HW, Sorensen HT, Hallas J. The Danish National Prescription Registry. Scand J Public Health 2011;39:38-41.

18. WHO. WHO Collaborating Centre for Drug Statistics Methodology (WHOCC). Oslo, Norway. Anatomical Therapeutic Chemical (ATC) classification system. 2011:2016. http://www.whocc.no/atc/ structure_and_principles/ (accessed online Nov 2016).

19. WHO. WHO Collaborating Centre for Drug Statistics Methodology (WHOCC). Oslo, Norway. Defined daily dose; ATC/DDD index, 2015. http://www.whocc.no/atc ddd index/

20. Andersen JS, Olivarius Nde F, Krasnik A. The Danish National Health Service Register. Scand J Public Health 2011;39:34-7.

21. Statistics Denmark, Copenhagen, Denmark. 2016. http://www.dst.dk/ en (accessed online November 2016).

22. Mors O, Perto GP, Mortensen PB. The Danish Psychiatric Central Research Register. Scand J Public Health 2011;39:54-7.

23. Chee CY, Chong YS, Ng TP, et al. The association between maternal depression and frequent non-routine visits to the infant's doctor-a cohort study. J Affect Disord 2008;107:247-53. 
24. Olesen C, Sondergaard C, Thrane N, et al. Do pregnant women report use of dispensed medications?. Epidemiology 2001;12:497-501.

25. Ehrenstein V. Association of Apgar scores with death and neurologic disability. Clin Epidemiol 2009;1:45-53.

26. Li J, Olsen J, Vestergaard M, et al. Low Apgar scores and risk of childhood attention deficit hyperactivity disorder. J Pediatr 2011;158:775-9.

27. Hirvonen $\mathrm{M}$, Ojala $\mathrm{R}$, Korhonen $\mathrm{P}$, et al. Cerebral palsy among children born moderately and late preterm. Pediatrics 2014;134:e1584-93.

28. Sun $Y$, Vestergaard M, Pedersen CB, et al. Apgar scores and long-term risk of epilepsy. Epidemiology 2006;17:296-301.

29. Ehrenstein V, Sorensen HT, Pedersen L, et al. Apgar score and hospitalization for epilepsy in childhood: a registry-based cohort study. BMC Public Health 2006;6:23.

30. Li J, Cnattingus S, Gissler M, et al. The 5-minute Apgar score as a predictor of childhood cancer: a population-based cohort study in five million children. BMJ Open 2012;2:10.
31. Glymour MM, Greenland S. Chapter 12: causal diagrams. In: Rothman KJ, Greenland S, Lash TL, eds. Modern epidemiology. Philadelphia, USA: Lippincott Williams \& Wilkins, 2008:183-209.

32. Li J, Yang H, Guldin MB, et al. Increased usage of primary healthcare in persons exposed to severe stress in prenatal life: a national population-based study in Denmark. BMJ Open 2015;5: e005657.

33. Bromley R, Weston J, Adab N, et al. Treatment for epilepsy in pregnancy: neurodevelopmental outcomes in the child. Cochrane Database Syst Rev 2014;10:CD010236.

34. Bromley R. The treatment of epilepsy in pregnancy: the neurodevelopmental risks associated with exposure to antiepileptic drugs. Reprod Toxicol 2016;64:203-10.

35. Banach R, Boskovic R, Einarson T, et al. Long-term developmental outcome of children of women with epilepsy, unexposed or exposed prenatally to antiepileptic drugs: a meta-analysis of cohort studies. Drug Saf 2010;33:73-9. 